\title{
IGuard: An Intelligent IOT based Security System for Server Rooms in Industries
}

\author{
N.K. Kadale ${ }^{1}$, Kiran Kokate ${ }^{2}$, Jay Patel ${ }^{3}$, Shashikant Mharasale ${ }^{4}$, Sagar Sawant ${ }^{5}$ \\ Professor, Computer Department, NBN Sinhgad School of Engineering, Pune ${ }^{1}$ \\ Student, Computer Department, NBN Sinhgad School of Engineering, Pune ${ }^{2}$
}

\begin{abstract}
Internet of Things (IoT) has provided a promising opportunity to build powerful industrial systems and applications by leveraging the growing and wireless, mobile, and sensor devices. A wide range of industrial IoT applications have been developed and deployed in recent years. In an effort to understand the development of IoT in industries, key enabling technologies, major IoT applications in industries, and identifies research Trends and challenges.
\end{abstract}

Keywords: RFID, WSN, Sensors.

\section{INTRODUCTION}

As recent technology, the Internet of Things (IoT) is expected to offer promising solutions to transform the operation and role of many existing industrial systems such as transportation systems and manufacturing systems. For example, when IoT is used for creating intelligent transportation systems, the transportation authority will be able to track each vehicle's existing location, monitor its movement, and predict its future location and possible road traffic. The term IoT was initially proposed to refer to uniquely identifiable interoperable connected objects with radio-frequency identification (RFID) technology.

With the advancements in Internet technologies and Wireless Sensor Networks (WSN), a new trend in the era of ubiquity is being realized. Enormous increase in users of Internet and modifications on the internetworking technologies enable networking of everyday objects. "Internet of Things (IoT)" is all about physical items talking to each other, machine-to-machine communications and person-to-computer communications will be extended to "things" Key technologies that will drive the future IoT will be related to Smart sensor technologies including WSN, Nanotechnology and Miniaturization. Key technologies that will drive the future IoT will be related to Smart sensor technologies including WSN, Nanotechnology and Miniaturization.

Humans usually inside their home interact with the environment settings like light, door, air, etc., and regulate accordingly. If the settings of the environment can be made to respond to human behaviour automatically, then there are several advantages. The automation of home settings to act according to the inhabitant requirements is termed as intelligent home automation system. Ambient intelligence responds to the behaviours of inhabitants in home and provides them with various facilities.

C The smart industries designed so far arefor different purposes such as information collection and decision support system for the wellbeing of the inhabitants.

Such technologies will act as a catalyst to the evolution of a new generation of services that will provide a great impact on the social and technological eco-system.

\section{SYSTEM ARCHITECTURE}

The server side architecture is primarily made up of a Model View Controller (MVC) framework. The framework provides access to the relational database server and other class objects within the server model. The database server is used to store merchant profile and other geographical related data. As it pertains to necessary information such as earth coordinates namely latitudes and longitudes, street address, zip codes of every advertisement is stored within it. This data is essentially used to retrieve the relevant data/information by comparing the user's geographical presence. The framework uses Active Records to make logical decisions. It also handles table associations, transactions, validations and relationship between objects and databases. The active record access data from database by retrieving and wrapping them into a class object

\section{CLIENT MODEL}

The client model mainly comprises of the client-user. The client model incorporates a mobile application program that handles all services such as requests from various tasks including application or program is needed to access GPS information at the client side. The existence of Location using various sensor will helps the developer to concentrate more on building the secure severs system within the client model. All the developer has to do is to check the data sense by the sensor to get user's current location by defining the appropriate sensor like light, humidity, temp, door etc. 


\section{AD LOCATION API MODULE}

The system architecture also includes a custom build API which acts like an interface between the server and client model. To sense is there anyone in the server room. API is made to run at the server side parallel to the central server program. It belongs to a class of web APIs known as Representation State Transfer APIs. REST APIs functions by using simple HTTP requests. The API is built using MVC framework similar to the server program. The API and the server program are both commonly connected to the server database. The API is delegated to perform necessary function such as retrieving information from the server database.

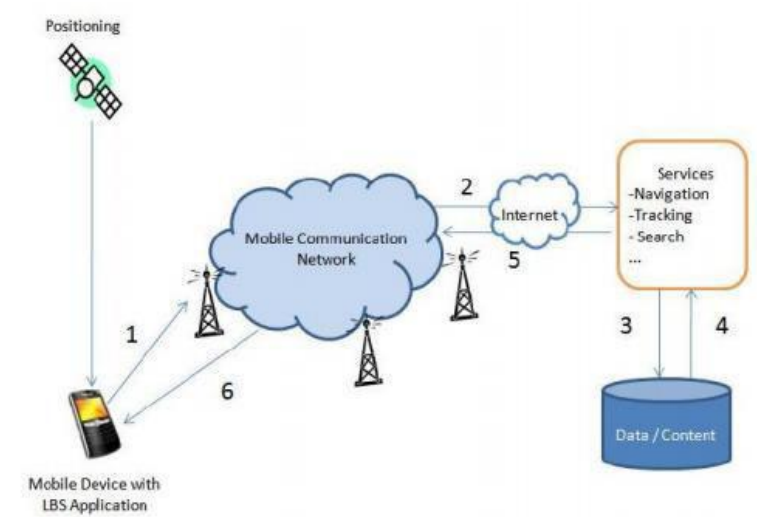

\section{CONCLUSION}

As a complex cyber-physical system, IoT integrates various devices equipped with sensing, identification, processing, com- mutilation, and networking capabilities. In particular, sensors and actuators are getting increasingly powerful, less expensive and smaller, which makes their use ubiquitous. To develop industrial applications such as automated monitoring, control, management, and maintenance.

Due to the rapid advances in technology and industrial infrastructure, IoT is expected to be widely applied to industries. For example, the food industry is integrating WSN and RFID to build automated systems for tracking, monitoring, and tracing food quality along the food supply chain in order to improve food quality.

This paper reviews the recent researches on IoT from the Industrial perspective. We firstly introduce the background and SOA models of IoT and then discuss the fundamental technologies that might be used in IoT. Next, we introduce some key industrial applications of IoT.

Afterward, we analysed the research challenges and future trends associated with IoT. Different from other IoT survey papers, a main contribution of this review paper is that it focuses on industrial IoT applications and highlights the challenges and possible research opportunities for future industrial researchers.

\section{REFERENCES}

[1]. D. Surie, O. Laguionie, T. Pederson, "Wireless sensor networking of everyday objects in a smart home environment", Proceedings of theInternational Conference on Intelligent Sensors, Sensor Networks and Information Processing-ISSNIP- 2008, pp. $189-$ 194.

[2]. Vision and Challenges for Realizing the Internet of Things, uropeanUnion 2010,ISBN 9789279150883.

[3]. Internet 3.0: The Internet of Things. (C) Analysys Mason Limited 2010.

[4]. M. Eisenhauer, P. Rosengren, P. Antolin, "A Development Platform for Integrating Wireless Devices and Sensors into Ambient Intelligence Systems", Proceedings of the 6th Annual IEEE Communications Society Sensor, Mesh and Ad Hoc Communications and Networks Workshops, SECON-2009, pp.1-3.

[5]. S. Hong, D. Kim, M. Ha, S. Bae, S. Park, W. Jung, J.E. Kim, "SNAIL: an IP-based wirelesssensor network approach to the internet of things", IEEE Wireless Communications,2010, Vol. 17, Issue.6, pp. $34-42$.

[6]. N.Bui, A.P.Castellani, P. Casari, M. Zorzi, "The internet of energy: a web-enabled smart grid system", IEEE Network, 2012, Vol.26, Issue. 4 , pp. $39-45$. 